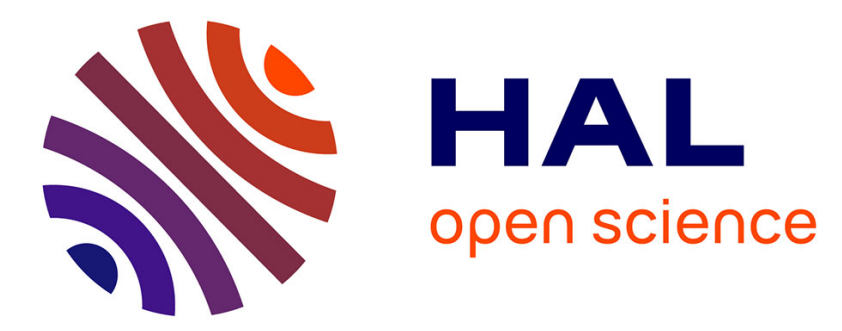

\title{
Ultra-high-Q whispering-gallery mode resonators in the pulsed-pump regime
}

Thomas Daugey, Jean-Marc Merolla, Yanne Chembo

\section{To cite this version:}

Thomas Daugey, Jean-Marc Merolla, Yanne Chembo. Ultra-high-Q whispering-gallery mode resonators in the pulsed-pump regime. Laser Resonators, Microresonators, and Beam Control XXII, Mar 2020, San Francisco, United States. pp.16, 10.1117/12.2544459 . hal-03014099

\section{HAL Id: hal-03014099 \\ https://hal.science/hal-03014099}

Submitted on 4 Dec 2020

HAL is a multi-disciplinary open access archive for the deposit and dissemination of scientific research documents, whether they are published or not. The documents may come from teaching and research institutions in France or abroad, or from public or private research centers.
L'archive ouverte pluridisciplinaire $\mathbf{H A L}$, est destinée au dépôt et à la diffusion de documents scientifiques de niveau recherche, publiés ou non, émanant des établissements d'enseignement et de recherche français ou étrangers, des laboratoires publics ou privés. 


\title{
Ultra-high-Q whispering-gallery mode resonators in the pulsed-pump regime
}

\author{
Thomas Daugey ${ }^{1}$, Jean-Marc Merolla ${ }^{1}$ and Yanne K. Chembo ${ }^{2}$ \\ ${ }^{1}$ FEMTO-ST Institute, CNRS and Univ. Bourgogne Franche-Comté, \\ 15B Avenue des Montboucons, 25030 Besançon cedex, France \\ ${ }^{2}$ Institute for Research in Electronics and Applied Physics (IREAP), \\ and Department of Electrical and Computer Engineering, \\ University of Maryland, 8279 Paint Branch Dr, College Park MD 20742, USA.
}

\begin{abstract}
We have developed an experimental setup in order to study whispering gallery mode resonators in the pulsedpump regime. Since the pulse repetition rate needs to match the free spectral range of the resonator, the pulse generator we have built is flexible in terms of both pulse repetition rate and pulsewidth. A rich variety of dynamical phenomena are observed in the system.
\end{abstract}

Keywords: Whispering gallery mode resonators, solitons, solitonic compression

\section{INTRODUCTION}

Whispering-gallery mode resonators are a versatile platform capable of hosting a wide diversity of dynamics (for a complete overview, see refs. $\left.{ }^{1-15}\right)$.

In the continuous pumped regime, it is possible to generate different patterns depending on the dispersion regime. In the anomalous dispersion regime, one can excite Turing rolls, chaotic dynamics, solitons, soliton molecules/crystals and breathers. ${ }^{14,15}$ Experimental work has been reported regarding soliton generation ${ }^{16,17}$ in WGMR in the literature. This work has been done exclusively in the continuous regime, meaning a continuous wave laser has been used to pump the WGMR.

Recently, work on the driving of WGMRs with a pulsed pump has been reported in ref. ${ }^{18}$ Driving a WGMR with a pulsed source, or equivalently, driving a frequency comb with a comb spacing matching (or very close to) the free spectral range of the resonator, could allow us to reach states different from the ones listed above. In this article we propose an architecture which can generates fairly high peak power pulses with flexible repetition rate, pulse duration and pulse state. By pumping a $\mathrm{MgF}_{2}$ WGMR, we observed the generation of asymmetric frequency combs when slightly tuning the repetition rate of the pulses.

We here report some results obtained experimentally, and provide a preliminary physical explanation regarding the various spectra that have been observed.

\section{THE SYSTEM}

The system is made of two subsystems. The first one is the pulse generator represented schematically on Fig. 1. Its architecture is similar to the optoelectronic oscillator presented in ref. ${ }^{19}$ A narrow linewidth $\mathrm{CW}$ laser is modulated in phase and in amplitude in order to generate chirped pulses. The pulses are then amplified and launched into an optical fiber where they undergo solitonic compression. At the output of the fiber we reach high-peak power, picoseconds pulses. Since we generate the pulses with optical modulators, we can freely choose the repetition rate of the pulses within their bandwidth and with $\mathrm{kHz}$ resolution.

The bottom left trace on Fig. 1 shows both the experimental and simulation average autocorrelation traces. We can see a fairly good agreement between both. The bottom right figure represents the pulse evolution as it

Further author information: (Send correspondence to Y. K. C.)

E-mail: ykchembo@umd.edu 

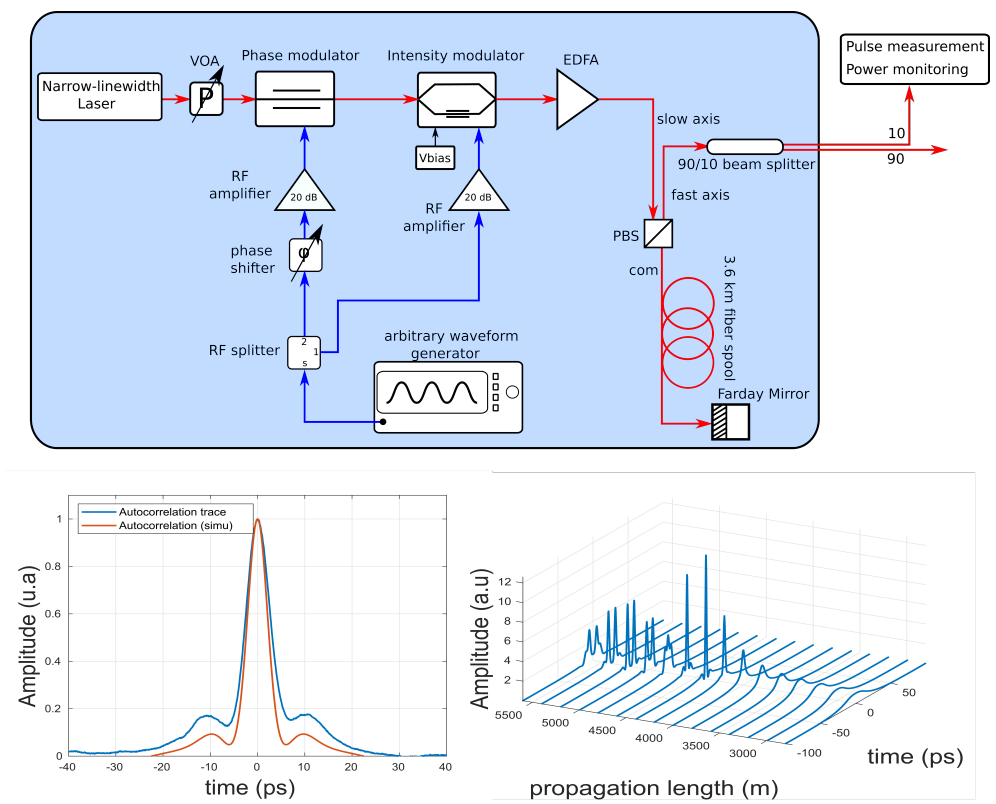

Figure 1. Top : Schematic representation of the experimental setup used to generate pulses with variable repetition rate. Left : Autocorrelation (experimental in blue and simulation in orange) of the pulses at the output of the pulse generator. Right : simulation of state of the pulse as a function propagation length.

propagates through the fiber and shows the versatility of our system. By changing the length of the fiber spool we can change the pulse duration and peak power until we reach the maximum compression rate (highest peak on the figure) or we can even go further and generate dual peak states.

The second system is the WGMR itself as well as the taper fiber used to couple light into it.

In order to lock the laser pump laser onto a resonance of the WGMR we use the Pound-Drever-Hall (PDH) method which requires the use of a phase modulator and an photodiode. However, the PDH lock can be perturbed if an other source of light reaches the photodiode, or if the intracavity power induces thermal instabilities. ${ }^{20}$ This is why we have implemented a double circulator architecture visible on figure 2 . This provides two optical paths, one which goes from the phase modulator through the WGMR and ends at the photodiode, and the other one which starts from the pulse generator, goes through the WGMR and ends up in the port 3 of the first circulator. With this setup, the stability of the lock is totally independent from the power of the pulses at the output of the pulse generator.

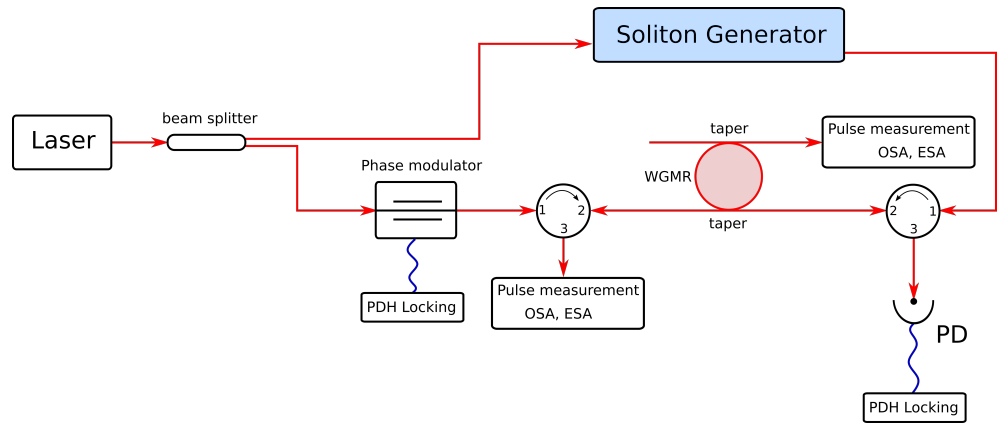

Figure 2. Schematic representation of the complete experimental setup used to drive the WGMR in the pulsed pump regime. The blue box labeled "Soliton generator" is the system represented on figure 1. 


\section{RESULTS AND DISCUSSION}

When we first drove the WGMR with the pulses tuned at a repetition rate matching the FSR of the particular resonance we were locked on we observed a broadening of the spectrum at the output of the WGMR relative to the input. This meant that the pulses were coupled into the resonator and the incoming frequency comb was broadened via four-wave mixing.

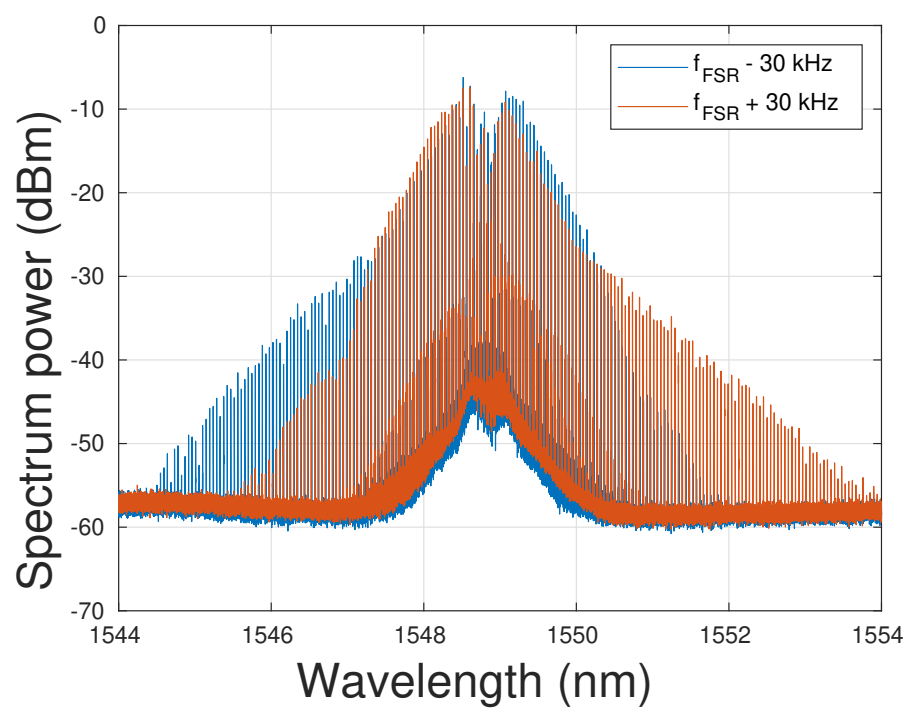

Figure 3. Spectra at the ouptut of a $\mathrm{MgF}_{2}$ WGMR when the repetition rate of the pulse generator is slighly detuned (few tens of $\mathrm{kHz}$ ) relative to the FSR of the resonances. We observe a broadening of the spectrum toward the higher frequency when the repetition rate is lower than the FSR and toward the lower frequency when the repetition rate is higher than the FSR.

We then varied a few parameters such as the input power or the polarization and observed the change in dynamics at the output of the WGMR. The dynamics of the system was drastically modified as we changed the modulation frequency applied on the modulators of the pulse generator, that is, the repetition rate of the pulses coupled into the resonator. When slightly changing the repetition rate (a few tens of kilohertz relative to the FSR of the resonance), we observed an asymmetric broadening of the frequency comb. As we can see on Fig. 3, the spectrum broadens toward the higher wavelength or the lower frequency when the repetition rate is increased relative to the FSR of the resonance and is broadens toward the lower wavelength or higher frequency when the repetition rate is decreased relative to the FSR of the resonance.

Since we use a frequency comb as the drive of the resonator, we do not couple light in the resonance on which the laser is locked only but in many resonances of the same family, each of them separated by one FSR from its neighbor. In order to provide an explanation for the phenomenon we have observed, we need to picture position of the frequency comb used as the pump relative to the family of resonance. When the repetition rate of the pump is increased, the frequency comb broadens. On the right of the resonance the laser is locked on, the frequency comb be shifted toward the blue side of the resonances. Similarly, the comb will be shifted toward the red side of the resonances on the left side of the central resonance. The reverse is true when the the repetition rate of the pump is decreased. If we link the explanations above to the experimental spectra, we observe that on the broadening of the frequency inside the resonator is observable only on the side where the pulses are red detuned relative to the center of the resonances.

It was found that in order to generate solitons in a WGMR, red detuning the pump relative to the center of the resonance seemed to be the most preferable condition. 


\section{CONCLUSION}

We developed a versatile and flexible pulse generator where both the peak power and the repetition rate of the pulses can be easily tuned. We than used this pulse generator to pump a WGMR, and analyze the mismatch with regard to the FSR of the resonance on which we are locked. We have observed asymmetric frequency comb generation when driving a resonator in the pulsed pump regime. Future work will explore this dynamical regime with further detail. Specific efforts will also be devoted to the understanding of the interplay of these combs with Raman ${ }^{21-26}$ and Brillouin nonlinearities, ${ }^{27-30}$ as well as possible applications in metrology. ${ }^{31,32}$

\section{REFERENCES}

1. K. Vahala, "Optical microcavities," Nature 424, 839-846 (2003).

2. A. B. Matsko, A A. Savchenkov, D. Strekalov, V. S. Ilchenko, and L. Maleki, "Review of applications of whispering-gallery mode resonators in photonics and nonlinear optics," IPN Progress Report, 42-162 (2005).

3. A. B. Matsko and V. S. Ilchenko, "Optical resonators with whispering gallery modes - Part I: basics," IEEE J. Sel. Top. Quantum Electron. 12, 3-14 (2006).

4. V. S. Ilchenko and A. B. Matsko, "Optical resonators with whispering gallery modes - Part II: applications," IEEE J. Sel. Top. Quantum Electron. 12, 15-32 (2006).

5. P. DelHaye, A. Schliesser, O. Arcizet, T. Wilken, R. Holzwarth, and T. J. Kippenberg, Optical frequency comb generation from a monolithic microresonator, Nature 450, 1214-1217 (2007).

6. A. Chiasera, Y. Dumeige, P. Féron, M. Ferrari, Y. Jestin, G. N. Conti, S. Pelli, S. Soria, and G. C. Righini, "Spherical whispering-gallery-mode microresonators," Laser Photon. Rev. 4, 457-482 (2010).

7. G. Kozyreff, J. L. Dominguez-Juarez, and J. Martorell, Nonlinear optics in spheres: from second harmonic scattering to quasi-phase matched generation in whispering gallery modes, Laser Photonics Rev. 5, 737-749 (2011).

8. D. J. Moss, R. Morandotti, A. L. Gaeta, and M. Lipson, New CMOS-compatible platforms based on silicon nitride and Hydex for nonlinear optics, Nat. Photonics 7, 597, (2013).

9. A. Coillet et al., Microwave Photonics Systems Based on Whispering-gallery-mode Resonators, J. Vis. Exp. 78, e50423 (2013).

10. W. Liang, A. A. Savchenkov, Z. Xie, J. F. McMillan, J. Burkhart, V. S. Ilchenko, C. W. Wong, A. B. Matsko, and L. Maleki, Miniature multioctave light source based on a monolithic microcavity, Optica 2, 40-47 (2015).

11. G. Lin, S. Diallo, J. M. Dudley, and Y. K. Chembo, Universal nonlinear scattering in ultra-high Q whispering gallery-mode resonators, Opt. Express 24, 14880 (2016).

12. D. V. Strekalov, C. Marquardt, A. B Matsko, H. G. L. Schwefel, and G. Leuchs, "Nonlinear and quantum optics with whispering gallery resonators," J. Opt. 18, 123002 (2016).

13. I. Breunig, Three-wave mixing in whispering gallery resonators, Laser Photonics Rev. 10, 569-587 (2016).

14. G. Lin, A. Coillet, and Y. K. Chembo, Nonlinear photonics with high-Q whispering-gallery mode resonators, Adv. Opt. Photon. 9, 828-890 (2017).

15. A. Pasquazi et al., Micro-combs: a novel generation of optical sources, Phys. Rep. 729, 1-81 (2018).

16. T. Herr, V. Brasch, J. Jost, C. Wang, N. Kondratiev, M L. Gorodetsky, and T. J. Kippenberg, Temporal solitons in optical microresonators, Nature Photonics 8, 145-152 (2014).

17. T. J. Kippenberg, A. L. Gaeta, M. Lipson, and M. L. Gorodetsky, Dissipative Kerr solitons in optical microresonators, Science 361, eaan8083 (2018).

18. M. Anderson,R. Bouchand, E. Obrzud, J. Liu, S. Karlen, S. Lecomte, T. Herr, and T. J. Kippenberg, Broadband Efficient Soliton Microcombs in Pulse-Driven Photonic Microresonators, OSA, CLEO: Science and Innovations, 2019.

19. Y. K. Chembo, A. Hmima, P-A. Lacourt, L. Larger, and J. M. Dudley, Generation of ultralow jitter optical pulses using optoelectronic oscillators with time-lens soliton-assisted compression, IEEE J. Lightw. Technol. 27, 5160-5167 (2009).

20. S. Diallo, G. Lin, and Y. K. Chembo, Giant thermo-optical relaxation oscillations in millimeter-size whispering gallery mode disk resonators, Opt. Lett. 40, 3834-3837 (2015). 
21. T. J. Kippenberg, S. M. Spillane, D. K. Armani and K. J. Vahala "Ultralow-threshold microcavity Raman laser on a microelectronic chip" Opt. Lett. 29, 1224-1227 (2004).

22. I. S. Grudinin and L. Maleki, Ultralow-threshold Raman lasing with $\mathrm{CaF}_{2}$ resonators, Opt. Lett. 32, 166-168 (2007).

23. D. Farnesi, F. Cosi, C. Trono, G.C. Righini, G.N. Conti, and S. Soria, Stimulated anti-Stokes Raman scattering resonantly enhanced in silica microspheres, Opt. Lett. 39, 5993-5996 (2014).

24. A. Chen-Jinnai, T. Kato, S. Fujii, T. Nagano, T. Kobatake, and T. Tanabe, Broad bandwidth thirdharmonic generation via four-wave mixing and stimulated Raman scattering in a microcavity, Opt. Express 24, 26322-26331 (2016).

25. G. Lin and Y. K. Chembo, Phase-locking transition in Raman combs generated with whispering gallery mode resonators, Optics Lett. 41, 3718-3721 (2016).

26. M. Karpov, H. Guo, A. Kordts, V. Brasch, M. H. P. Pfeiffer, M. Zervas, M. Geiselmann, and T. J. Kippenberg, Raman self-frequency shift of dissipative Kerr solitons in an optical microresonator, Phys. Rev. Lett. 116, 103902 (2016).

27. J. Li, H. Lee, and K. J. Vahala, Microwave synthesizer using an on-chip Brillouin oscillator, Nat. Commun. 4, 2097 (2013).

28. I. S. Grudinin, A. B. Matsko, and L. Maleki, Brillouin lasing with a $\mathrm{CaF}_{2}$ whispering gallery mode resonator, Phys. Rev. Lett. 102, 043902 (2009).

29. C. Guo, K. Che, P. Zhang, J. Wu, Y. Huang, H. Xu, and Z. Cai, Low-threshold stimulated Brillouin scattering in high- $Q$ whispering gallery mode tellurite microspheres, Opt. Express 23, 32261 (2015).

30. W. Loh, J. Becker, D. C. Cole, A. Coillet, F. N. Baynes, S. B. Papp, and S. A. Diddams, A microrodresonator Brillouin laser with $240 \mathrm{~Hz}$ absolute linewidth, New J. Phys. 18, 45001 (2016).

31. K. Saleh, G. Lin, and Y. K. Chembo, Effect of laser coupling and active stabilization on the phase noise performance of optoelectronic microwave oscillators based on whispering-gallery-mode resonators, IEEE Phot. J. 7, 1-11 (2014).

32. K. Saleh and Y. K. Chembo, On the phase noise performance of microwave and millimeter-wave signals generated with versatile Kerr optical frequency combs, Optics express 24, 25043-25056 (2016). 